\title{
Estudiantes universitarios descubren redes sociales y edublog como medio de aprendizaje
}

\section{(University students discover social networks and edublog as a learning tool)}

\author{
Carolina González-Hernando \\ Lorena Valdivieso-León \\ Verónica Velasco-González \\ Universidad de Valladolid, UVa (España)
}

DOI: http://dx.doi.org/10.5944/ried.23.1.24213

\section{Cómo referenciar este artículo:}

Gonzalez-Hernando, C., Valdivieso-León, L., y Velasco-González, V. (2020). Estudiantes universitarios descubren redes sociales y edublog como medio de aprendizaje. RIED. Revista Iberoamericana de Educación a Distancia, 23(1), pp. 223-239. doi: http://dx.doi.org/10.5944/ried.23.1.24213

\section{Resumen}

Entre los desafíos considerados fáciles de abordar según the NMC Horizon Report 2018, están la alfabetización digital y la combinación del aprendizaje formal e informal. Los universitarios pertenecen en su mayoría a las generaciones Millennials y Z, y se les presupone mayor capacidad de autoaprendizaje desde internet. Se pretende identificar el perfil de uso de internet de los estudiantes de enfermería nativos digitales y grado de satisfacción que genera la utilización de un blog educativo. Se administró un cuestionario anónimo y voluntario a estudiantes de $1^{\mathrm{O}}$ y $2^{\circ}$ curso del Grado en Enfermería de la Universidad de Valladolid durante el curso académico 2017-18. Se trata de un estudio descriptivo de corte transversal. Participaron el 91,6 \% (n=225) de los estudiantes matriculados. El 74,7 \% no conocía LinkedIn. Google+ fue la herramienta de mayor uso académico, seguida de Twitter. Más de un 80 \% tenía conocimiento de nivel medio de Facebook, Twitter, Pinterest, Instagram y Moodle. Destaca el conocimiento avanzado que poseen de Instagram. Un $48 \%$ tenían conocimientos nulos o bajos sobre el uso de blogs. La satisfacción ante la utilización de Edublog Enfermería obtuvo una puntuación global de $8,04( \pm 1,37)$ sobre 10 . Los estudiantes utilizan las redes sociales como medio de comunicación y no con fines académicos. Los escasos conocimientos sobre el uso de los blogs y la elevada satisfacción que reportó su uso, indica la necesidad de reforzar estas herramientas. Los blogs y redes sociales pueden ser útiles para dotar de competencias digitales a los estudiantes de enfermería logrando un aprendizaje continuo y actualizado. 
Palabras clave: aprendizaje a través de TIC; aprendizaje electrónico; edublog; enfermería; enseñanza y formación.

\begin{abstract}
Among the challenges considered easy to address according to the NMC Horizon Report 2018 are digital literacy and the combination of formal and informal learning. The university students belong mostly to the Millennials and $\mathrm{Z}$ generations, and they are supposed to have a greater capacity for self-learning from the Internet. The aim is intended to identify the profile of internet use by digital native nursing students and degree of satisfaction generated by the use of an educational blog. An anonymous and voluntary questionnaire was administered to 1st and 2nd year students of the Degree in Nursing of the University of Valladolid during the 2017-18 academic year. It is a descriptive cross-sectional study. 91,6\% ( $=225)$ of the enrolled students participated. 74,7 \% did not know LinkedIn. Google+ was the most widely-used academic tool, followed by Twitter. More than $80 \%$ had mid-level knowledge of Facebook, Twitter, Pinterest, Instagram and Moodle. The advanced knowledge possessed about Instagram stands out. $48 \%$ had no or low knowledge about using blogs. The satisfaction of the students who used this educational blog Edublog Enfermería earned an overall score of $8,04( \pm 1,37)$ out of 10 . Students use social networks as a means of communication and not for academic purposes. The scarce knowledge about the use of blogs and the high satisfaction reported about their use indicates the need to strengthen these tools. Blogs and social networks can be useful to provide digital to nursing students achieving continuous and updated learning.
\end{abstract}

Keywords: ICT based learning; e-learning training; edublog; nursing; teaching and training.

La aparición de Internet y de las redes sociales, ha cambiado la forma de comunicarnos y de interactuar. Hoy en día la tecnología permite comunicarnos en cualquier momento y desde cualquier lugar. Los medios sociales digitales como Facebook, Twitter, YouTube y los blogs impregnan nuestras vidas (Carroll, Bruno, y vonTschudi, 2016).

Las nuevas generaciones de jóvenes que ingresan en los programas de estudios universitarios están cambiando sensiblemente, ello hace necesario que seamos conscientes de que buena parte de las transformaciones de las universidades del siglo XXI deben adaptarse a estos rápidos cambios (Ortega y Soto, 2016).

Según la Real Academia Española (2016) una generación es "el conjunto de personas que, habiendo nacido en fechas próximas y recibido educación e influjos culturales y sociales semejantes, adoptan una actitud en cierto modo común en el ámbito del pensamiento o de la creación".

Aunque hay diferentes clasificaciones de generaciones, en el mundo educativo y laboral se diferencian las siguientes: Generación Baby-Boomers (nacidos entre 1946-1964), Generación X (nacidos entre 1965-1979), Generación Y o Millennials 
(nacidos entre 1980-1999), Generación Z (nacidos entre 2000-2010) y Generación Alpha/Glass (nacidos desde 2010).

Las generaciones $\mathrm{Y}$ y $\mathrm{Z}$ son las que corresponden actualmente a la mayoría de nuestros estudiantes universitarios de enfermería. En España, el contacto con dispositivos móviles, así como el uso de internet, fue más tardío en los "Millennials" o Generación Y en comparación con la Generación Z, en la que Internet ha estado desde las fases más tempranas de su socialización y educación (Begazo y Fernández, 2015). Los primeros, crecieron rodeados de tecnología caracterizándose por el dominio digital, ser sociales, críticos y exigentes, app adictos y por utilizar varias pantallas a la vez (Bernal, 2015). La sobre estimulación en la que están inmersos implica una atención simultánea a varios asuntos, un aprendizaje más rápido si les resulta interesante, son muy independientes y dan como válida toda la información que les llega de internet y de las redes sociales, son dependientes de la tecnología (Bongarrá, 2011).

Los jóvenes de la generación $\mathrm{Z}$ nacieron con los dispositivos móviles e internet siendo estos imprescindibles para su vida. Son digitales muy adaptables, multipantalla y multidispositivos (hasta cinco pantallas), los caracteriza la rapidez e impaciencia, ser individualistas, interactivos y que se manejan muy bien en las comunidades globales (Net-Learning, 2016). No creen mucho en la educación formal porque la plantean desde otra perspectiva ya que gran parte del aprendizaje lo realizan fuera del aula, aprenden en red y desde la red usando YouTube o buscando información online, y desean mayor grado de personalización. En esta generación emerge la conciencia social y el espíritu crítico (Verdú, 2015).

Aunque puede pensarse que los estudiantes actuales están alfabetizados digitalmente, la investigación muestra que en el contexto educativo hay que proporcionarles habilidades necesarias para que sean productivos en el mundo laboral cambiante, siendo ésta una prioridad para las universidades. Los desafíos en la adopción de tecnologías en Educación Superior que se consideran fáciles de abordar, según The NMC Horizon Report 2018 Higher Education Edition (Adams, Brown, Dahlstrom, Davis, DePaul, Diaz y Pomerantz, 2018), son la combinación del aprendizaje formal e informal (los video-tutoriales, el contenido en abierto y las redes sociales que permiten aprender en cualquier momento y en cualquier lugar) (Johnson, Adams, Cummins, Estrada, Freeman, y Hall, 2016). Según este informe, a corto plazo hay que impulsar la adopción de la tecnología en educación superior con el rediseño de espacios de aprendizaje, la combinación del aprendizaje formal e informal y la alfabetización digital, la cual fue definida por la American Library Association como "la habilidad de usar las tecnologías de la información y la comunicación para encontrar, comprender, valorar, crear y transmitir información digital, que requiere tanto competencias cognitivas como técnicas" (2013, p.2).

En la Educación de Ciencias de la Salud existe un desfase entre las competencias de los actuales y futuros profesionales y las necesidades de los pacientes, un trabajo en equipo que presenta deficiencias, enfoques técnicos a veces sin comprensión 
conceptual, orientación curativa en lugar de preventiva y un débil liderazgo (Frenk y cols., 2010).

Los pacientes cada vez más, recurren a Internet para consultar sobre salud y enfermedad. Según el informe del Observatorio Nacional de Telecomunicaciones de la Sociedad de la Información, el 96,0 \% utiliza Internet para consultar sobre salud, el 91,6 \% confía mucho en su médico y en su enfermera lo que refleja la responsabilidad de la capacitación digital del profesional sanitario (ONTSI, 2016).

En la actualidad, las enfermeras y enfermeros intervienen en blogs, redes sociales, seleccionan recursos online para sus pacientes generando la necesidad de desarrollar estas competencias profesionales desde las universidades (Kung, 2014). Enfermería se muestra como una de las profesiones sanitarias más activas, no solo dentro del hospital o centro de salud, sino en la Web 2.o. Ya sea a través de Twitter, Facebook, YouTube o blogs personales, se han convertido en las caras de la profesión enfermera, aunque sean virtuales (Redacción Médica, 2016).

Según el profesor Mayol (2016) "los profesionales sanitarios ven cómo su papel central como proveedores de servicios sanitarios se ve desplazado por el de gestores de información y conocimiento para los pacientes”.

El blog es una publicación en red que permite crear y editar contenido con mínimos conocimientos técnicos. Los blogs educativos o edublogs son útiles recursos pedagógicos que facilitan el proceso de enseñanza y aprendizaje y mejoran la comunicación con los nativos digitales, los estudiantes son más conscientes de su proceso de aprendizaje, aumenta la participación y el aprendizaje social (Molona, Valenciano y Valencia-Peris, 2015) y pueden ser motivadores (Aguaded, López y Alonso, 2010). Los profesores con el uso del blog cuentan con un formato para organizar materiales, integrar contenidos, insertar videos, enlaces y motivar con feedback. Los estudiantes de enfermería y de otras disciplinas, pueden mejorar sus capacidades de comunicación, aprendizaje colaborativo, de síntesis y de gestión de la información con estas herramientas innovadoras (Grassley y Bartoletti, 2009; Magg, 2005). Algunos docentes utilizan los blogs como recurso de apoyo complementario a la clase presencial.

Los medios sociales digitales y el entramado en red que estos posibilitan han permitido que entremos en una dinámica de aprendizaje colaborativo y de creación de contenidos. Muchos investigadores y docentes utilizan las redes sociales para compartir resultados de proyectos, reflexiones, artículos que favorecen la difusión de la ciencia (García-Álvarez de Toledo y Fernández-Sánchez, 2011).

Sin embargo, pese a su enorme impacto la investigación respecto al uso de los blogs educativos como medio de formación en enfermería aún es escasa (Papastavro, Hamari, Fuster, Istomina, y Salminen, 2016) y la capacitación de los docentes enfermeros en esta materia es dispar según la European Federation of Nurse Educators (FINE- Europe, 2016). 


\section{METODOLOGÍA}

En esta sección se desarrolla el abordaje metodológico de un estudio realizado por los autores cuyos objetivos fueron, en primer lugar, identificar cómo los estudiantes de enfermería utilizan las redes sociales y los blogs con fines académicos y sociales con el fin de hacer propuestas de mejora para el cambio educativo, y en segundo lugar, conocer el grado de satisfacción de los estudiantes en relación con la efectividad y el funcionamiento de un blog creado específicamente por sus profesores como herramienta educativa.

Los participantes del estudio fueron los estudiantes matriculados en los cursos de $1^{\circ}$ y $2^{\circ}$ de la Facultad de Enfermería de la Universidad de Valladolid durante el año académico 2017-18, de la generación Y y Z. Para la consecución de los objetivos se decidió llevar a cabo un estudio de tipo descriptivo y de corte transversal.

Se diseñó el "Edublog Enfermería" por cuatro profesores de la Facultad de Enfermería de la Universidad de Valladolid. Según Borrás (2015) el edublog es una herramienta de la Web 2.0 que pasa de ser sólo un repositorio o fuente de información, a ser una plataforma de trabajo colaborativo, en la que además de compartir información existe interacción. Más que una tecnología, representa una actitud de los usuarios, tanto productores como consumidores de la información. Permite la democratización de la producción y acceso a la información para la enseñanza-aprendizaje. El edublog se incorporó como herramienta educativa de la asignatura Salud Sexual y Reproductiva, impartida en el $2^{\circ}$ curso, después de haber finalizado el semestre académico, previa validación en un grupo de estudiantes con las mismas características, con acceso a través de la siguiente página web: https:// enfermeriauva.blogspot.com/

Se administró un cuestionario “ad hoc” anónimo, que se distribuye en tres apartados: datos sociodemográficos, datos sobre los conocimientos internautas y de redes sociales; y grado de satisfacción en relación con la efectividad y el funcionamiento del blog creado para los estudiantes de $2^{\circ}$ curso. Posteriormente, se administró el mismo cuestionario a los estudiantes de $1^{\circ}$ curso; excluyendo el tercer apartado porque estos estudiantes no utilizaron el blog, aunque tendrían acceso a este en el siguiente curso.

Se recogió información de las siguientes variables: edad, sexo, forma de acceso a la universidad, frecuencia de uso de internet, lugar y dispositivo de conexión, grado de conocimiento de Facebook, Twitter, de Google+, LinkedIn, Pinterest, Instagram, Moodle, correo electrónico, navegación por internet, YouTube, Google apps, Smartphone, uso de blogs para aumentar sus conocimientos y su grado de satisfacción en concreto con "Edublog Enfermería".

Posteriormente, los datos fueron almacenados y analizados con el paquete estadístico IBM SPSS V.23. Por un lado, las variables cualitativas (dicotómicas y politómicas) o cuasi-cuantitativas de escala ordinal se presentan en porcentajes, y por otro lado, las variables de respuesta continua con una escala Likert con valores 
del 1 al 10, para evaluar la satisfacción de la utilización del "Edublog Enfermería" que se expresa en medias y desviación estándar (DE).

\section{RESULTADOS}

Se obtuvo una participación del 91,6\% de los estudiantes matriculados en el curso de primero y/o segundo del Grado de Enfermería de la Universidad de Valladolid. Cuatro de los participantes fueron excluidos por tener edades superiores a 35 años. Por lo tanto, la muestra objeto de estudio estuvo compuesta por 225 estudiantes. La media de edad fue 19,4 años ( $\mathrm{DE} \pm 1,7)$, y el rango 18-33 años. El 8o \% ( $\mathrm{n}=180)$ fueron mujeres frente al 19,1 \% $(n=43)$ que fueron hombres. Los estudiantes de la muestra accedieron a la universidad a través de bachillerato en un $78,2 \%(n=176)$ (tabla 1).

Tabla 1. Características sociodemográficas de la muestra

\begin{tabular}{|l|l|r|r|}
\hline \multirow{4}{*}{ Sexo } & & $\mathrm{n}$ & $\%$ \\
\hline \multirow{5}{*}{ Forma de acceso universidad } & Hombre & 43 & 19,1 \\
\cline { 2 - 4 } & Mujer & 180 & 80,0 \\
\cline { 2 - 4 } & No contesta & 2 & 0,9 \\
\cline { 2 - 4 } & Bachillerato +PAU* & 176 & 78,2 \\
\cline { 2 - 4 } & Ciclo Superior+ PAU* & 43 & 19,1 \\
\cline { 2 - 4 } & Titulados & 1 & 0,4 \\
\cline { 2 - 4 } & Deportistas & 1 & 1,8 \\
\cline { 2 - 4 } & No contesta & 1 & 0,4 \\
\hline
\end{tabular}

*PAU-Prueba de acceso a la universidad

El 61,3 \% de los estudiantes utilizaba blogs o redes sociales para la enseñanza o aprendizaje, y un 43,1 \% indicó su utilidad como herramienta educativa. Un 95,6 \% se conectaba varias veces al día a internet. El 98,7 \% desde sus casas y el 89,3\% desde las instalaciones de la universidad. Los dispositivos más frecuentes utilizados para ello fueron el smartphone (96,4\%) y el ordenador portátil (83,1 \%) (ver tabla 2). 
Tabla 2. Perfil del conocimiento internauta de la muestra

\begin{tabular}{|c|c|c|c|}
\hline Estudiantes $(n=225)$ & & $\mathrm{n}$ & $\%$ \\
\hline \multirow{3}{*}{ Frecuencia uso internet } & Varias veces al día & 215 & 95,6 \\
\hline & Una vez al día & 9 & 4 \\
\hline & Varios días por semana & 1 & 0,4 \\
\hline \multirow{2}{*}{ Lugar conexión ${ }^{a}$} & Casa & 222 & 98,7 \\
\hline & Universidad & 201 & 89,3 \\
\hline \multirow{4}{*}{ Dispositivo conexión ${ }^{\mathrm{b}}$} & Ordenador de sobremesa & 70 & 31,1 \\
\hline & Portátil & 187 & 83,1 \\
\hline & Tablet & 76 & 33,8 \\
\hline & Smartphone & 217 & 96,4 \\
\hline \multirow{4}{*}{$\begin{array}{l}\text { Blogs o redes sociales para } \\
\text { enseñanza o aprendizaje }\end{array}$} & Uso & 138 & 61,3 \\
\hline & Utilidad (Si) & 97 & 43,1 \\
\hline & Utilidad (Indiferente) & 13 & 5,8 \\
\hline & Utilidad (No contesta) & 115 & 51,1 \\
\hline
\end{tabular}

${ }^{a}$ La conexión puede ser tanto en casa como en la universidad, se pueden elegir las dos opciones.

${ }^{\mathrm{b}}$ Un alumno puede utilizar varios dispositivos de conexión.

El 74,7 \% refirió no conocer LinkedIn. Google+ fue la fuente de acceso a la información que mayor uso académico obtuvo con un $25,3 \%$, seguida de Twitter con un $15,1 \%$. Más de un 80 \% tenía conocimiento de nivel medio de Facebook, Twitter, Pinterest, Instagram y Moodle. Instagram fue la red social con mayor conocimiento avanzado por parte de los estudiantes $8 \%$ y Google+ con una mayor proporción del $20 \%$ de la muestra con conocimientos básicos (ver tabla 3).

Más de un $60 \%$ de los estudiantes tenían conocimientos de nivel medio del correo electrónico, navegar por internet, YouTube, Google apps, Smartphones, aplicaciones en red y Moodle. Siendo en más de un $30 \%$ avanzados en el correo electrónico, navegar por internet y el uso de los smartphones. Cabe destacar que el $20 \%$ de los estudiantes, tenían conocimientos nulos o muy bajos sobre el uso de los blogs y el $28 \%$ conocimientos bajos (ver tabla 3 ). 
Tabla 3. Perfil del uso de redes sociales, internet y blogs de los estudiantes a estudio

\begin{tabular}{|c|c|c|c|c|c|c|c|c|c|c|c|}
\hline \multirow{2}{*}{\multicolumn{2}{|c|}{ Estudiantes $(n=225)$}} & \multicolumn{2}{|c|}{ Facebook } & \multicolumn{2}{|c|}{ Twitter } & \multicolumn{2}{|c|}{ Google+ } & \multicolumn{2}{|c|}{ Pinterest } & \multicolumn{2}{|c|}{ Instagram } \\
\hline & & $\mathrm{n}$ & $\%$ & $\mathrm{n}$ & $\%$ & $\mathrm{n}$ & $\%$ & $\mathrm{n}$ & $\%$ & $\mathrm{~N}$ & $\%$ \\
\hline \multirow{3}{*}{ Conocimiento } & Básico & 33 & 14,7 & 26 & 11,6 & 45 & 20 & 5 & 2,2 & 21 & 9,3 \\
\hline & Medio & 182 & 80,9 & 184 & 81,7 & 165 & 73,3 & 219 & 97,4 & 186 & 82,7 \\
\hline & Avanzado & 10 & 4,4 & 15 & 6,7 & 15 & 6,7 & 1 & 0,4 & 18 & 8 \\
\hline \multirow{2}{*}{ Uso } & Social & 159 & 70,7 & 164 & 72,9 & 3 & 1,3 & 2 & 0,9 & 147 & 65,3 \\
\hline & Académico & 24 & 10,7 & 34 & 15,1 & 57 & 25,3 & 2 & 0,9 & 3 & 1,3 \\
\hline
\end{tabular}

\begin{tabular}{|c|c|c|c|c|c|c|c|c|c|}
\hline \multirow{2}{*}{\multicolumn{2}{|c|}{ Estudiantes $(n=225)$}} & \multicolumn{2}{|c|}{$\begin{array}{c}\text { Correo } \\
\text { electrónico } \\
\end{array}$} & \multicolumn{2}{|c|}{$\begin{array}{c}\text { Navegar por } \\
\text { internet }\end{array}$} & \multicolumn{2}{|c|}{ Blog } & \multicolumn{2}{|c|}{ YouTube } \\
\hline & & $\mathrm{n}$ & $\%$ & $\mathrm{n}$ & $\%$ & $\mathrm{n}$ & $\%$ & $\mathrm{~N}$ & $\%$ \\
\hline \multirow{5}{*}{ Conocimiento } & Muy bajo & 0 & 0 & 1 & 0,4 & 45 & 20 & 0 & 0 \\
\hline & Bajo & 0 & 0 & 0 & 0 & 63 & 28 & 8 & 3,6 \\
\hline & Medio & 151 & 67,2 & 143 & 63,6 & 106 & 47,1 & 153 & 68 \\
\hline & Avanzado & 73 & 32,4 & 80 & 35,6 & 9 & 4 & 61 & 27,1 \\
\hline & No contesta & 1 & 0,4 & 1 & 0,4 & 2 & 0,9 & 3 & 1,3 \\
\hline
\end{tabular}

\begin{tabular}{|c|c|c|c|c|c|c|c|c|c|}
\hline \multirow{2}{*}{\multicolumn{2}{|c|}{ Estudiantes $(\mathrm{n}=225)$}} & \multicolumn{2}{|c|}{ Google apps } & \multicolumn{2}{|c|}{ Smartphones } & \multicolumn{2}{|c|}{$\begin{array}{c}\text { Aplicaciones } \\
\text { en red }\end{array}$} & \multicolumn{2}{|c|}{ Moodle } \\
\hline & & $\mathrm{n}$ & $\%$ & $\mathrm{n}$ & $\%$ & $\mathrm{n}$ & $\%$ & $\mathrm{n}$ & $\%$ \\
\hline \multirow{5}{*}{ Conocimiento } & Muy bajo & 12 & 5,3 & 3 & 1,3 & 5 & 2,2 & O & 0 \\
\hline & Bajo & 40 & 17,8 & 6 & 2,7 & 16 & 7,1 & 7 & 3,1 \\
\hline & Medio & 142 & 63,1 & 136 & 60,4 & 173 & 76,9 & 190 & 84,5 \\
\hline & Avanzado & 28 & 12,4 & 80 & 35,6 & 14 & 6,2 & 28 & 12,4 \\
\hline & No contesta & 3 & 1,3 & o & o & 17 & 7,6 & o & 0 \\
\hline
\end{tabular}

${ }^{a}$ La conexión puede ser tanto en casa como en la universidad, se pueden elegir las dos opciones.

${ }^{\mathrm{b}}$ Un alumno puede utilizar varios dispositivos de conexión.

Como se puede observar en la tabla 4, el grado de satisfacción del Edublog Enfermería, medida con una escala Likert con valores de respuesta de 1 a 10, de los estudiantes de $2^{0}$ curso fue de $7,98( \pm 1,61)$, siendo la evaluación global del blog de $8,04( \pm 1,37), 8,04( \pm 1,20)$ de su contenido, $8,04( \pm 1,31)$ de la utilidad del mismo como herramienta de aprendizaje, $7,43( \pm 1,64)$ de la resolución de dudas planteadas a lo largo del cuatrimestre, fueron resueltas para un $62,3 \%(n=71)$ de la muestra; y $8,24( \pm 1,44)$ del grado de recomendación a otros estudiantes.

En cuanto al uso del blog por parte de los estudiantes, 17,5 \% lo utilizó habitualmente, $79,8 \%$ a veces y únicamente un 2,6 \% no recurrió a él como herramienta educativa. 
Entre los contenidos del Edublog, los que más interés despertaron entre los estudiantes, fueron la sexualidad y la reproducción, con un $72,8 \%$ y un $43 \%$, respectivamente.

Tabla 4. Evaluación y grado de satisfacción de los estudiantes de $2^{\circ}$ curso respecto al Edublog Enfermería

\begin{tabular}{|c|c|c|c|}
\hline Estudiantes $2^{\circ}$ curso $(n=114)$ & & Media & $( \pm \mathrm{DE})$ \\
\hline \multirow{5}{*}{ Evaluación Edublog } & Global & 8,04 & 1,37 \\
\hline & Contenido & 8,04 & 1,20 \\
\hline & Utilidad & 8,04 & 1,31 \\
\hline & Resolución de dudas & 7,43 & 1,64 \\
\hline & Recomendación a otros estudiantes & 8,24 & 1,44 \\
\hline Grado satisfacción Edublog & & 7,98 & 1,61 \\
\hline Estudiantes $2^{\circ}$ curso $(n=114)$ & & $\mathrm{n}$ & $\%$ \\
\hline \multirow{3}{*}{ Uso del Edublog } & Sí, habitualmente & 20 & 17,5 \\
\hline & Sí, a veces & 91 & 79,8 \\
\hline & No & 3 & 2,6 \\
\hline \multicolumn{4}{|l|}{ Grado de interés en: } \\
\hline \multirow{3}{*}{ Sexualidad } & Sí & 83 & 72,8 \\
\hline & No & 26 & 22,8 \\
\hline & No contesta & 5 & 4,4 \\
\hline \multirow{3}{*}{ Reproducción } & Sí & 49 & 43 \\
\hline & No & 60 & 52,6 \\
\hline & No contesta & 5 & 4,4 \\
\hline \multirow{3}{*}{ Embriología } & Sí & 31 & 27,2 \\
\hline & No & 78 & 68,4 \\
\hline & No contesta & 5 & 4,4 \\
\hline \multirow{3}{*}{ Más enfermería } & Sí & 29 & 25,4 \\
\hline & No & 80 & 70,2 \\
\hline & No contesta & 5 & 4,4 \\
\hline \multirow{3}{*}{ Aprendizaje basado en problemas } & Sí & 27 & 23,7 \\
\hline & No & 82 & 71,9 \\
\hline & No contesta & 5 & 4,4 \\
\hline \multirow{3}{*}{ Resolución de dudas } & Sí & 71 & 62,3 \\
\hline & No & 39 & 34,2 \\
\hline & No contesta & 4 & 3,5 \\
\hline
\end{tabular}

DE: Desviación Estándar 


\section{DISCUSIÓN}

Los estudiantes de enfermería de la Universidad de Valladolid han cambiado y encontramos una brecha entre la forma de aprender de los "nativos digitales" y la forma de enseñanza. En las aulas se está produciendo un profundo cambio generacional en relación con el entorno tecnológico, diferenciándose los estudiantes de sus padres y profesores que son "inmigrantes digitales" (Prensky, 2001; Prensky, 2006).

Varios autores proponen que para reducir "brechas digitales y culturales" entre profesores y estudiantes y que mejoren sus relaciones interpersonales, hay que integrar la tecnología en las prácticas educativas y comunicativas. Para ello, los docentes deben disponer de alfabetización digital básica e integrarla en su práctica didáctica (Esteve, Gisbert, y Lázaro, 2016).

El uso de internet permite mayor flexibilidad para adaptarse al ritmo de aprendizaje de los estudiantes que suelen tener un perfil heterogéneo en cuanto a edad, madurez, experiencia de trabajo previo... (Bloomfield, Cornish, Parry, Pegram y Moore, 2013).

Hay que tener en cuenta que los jóvenes estudiantes son más autónomos, incrementan el autoaprendizaje y les es más fácil el aprendizaje online. Están hiperconectados a internet y lo usan sobre todo desde el teléfono móvil, aunque también desde el ordenador, tablet, aplicaciones y otros dispositivos (Dowling, 2015). Con los resultados de nuestra investigación, se comprueba que los estudiantes se sienten cómodos utilizando tecnologías digitales y aunque se conectan más desde sus hogares lo hacen también desde cualquier lugar. En la Era Digital el aprendizaje no se limita al aula, se expande y ya no existen barreras de espacio ni de tiempo, los contenidos permanecen accesibles optimizando el tiempo dedicado al estudio (Alonso, 2016; Mavrou y Loizou-Raouna, 2017).

Las redes sociales más utilizadas con una orientación social son Twitter y Facebook y suponen para los estudiantes, un medio de interacción para comunicarse con sus amigos y no tanto para fines académicos. Sin embargo, los medios sociales pueden ayudar en la educación y formación enfermera complementando los conocimientos tradicionales (Carroll y cols., 2016). Los medios sociales funcionan como una plataforma que puede apoyar a los profesores en la enseñanza de competencias comunicativas (gestión de información y escritura) y destacar la importancia de un trato de privacidad y ética con el paciente. Los estudiantes tienen conocimientos avanzados de Instagram que reflejan la importancia de la comunicación visual para estos jóvenes. Aunque existen barreras para integrar las redes sociales en la educación de enfermería, hay recursos como Facebook, Twitter o YouTube que se pueden utilizar con fines pedagógicos (Schmitt, Sims-Giddens, y Booth, 2012). Facebook posee grupos con intereses comunes para unirse, personas para compartir, un muro donde escribir opiniones, recomendar publicaciones, compartir imágenes, chat, servicio de mensajería. Twitter es otra de las redes cuyo éxito es la simplicidad, 
ambas se pueden utilizar con fines científicos y educativos (García-Álvarez de Toledo y Fernández-Sánchez, 2011). Existen experiencias de éxito con el uso en Facebook como la de Santillán, Cornejo, y Ausín (2012) quienes la utilizaron con fines docentes y de difusión científica en Enfermería.

La mayoría de los estudiantes desconocía LinkedIn, una red social gratuita que permite realizar una lista de habilidades que podría atraer a posibles empleadores, tener todos los contactos en un solo lugar, enviar mensajes, e informar de progresos en el perfil profesional (Citrome, 2015) y cada vez más, se utilizan estos medios para demostrar resultados de aprendizaje formal e informal (Johnson y cols., 2016).

En relación con los blogs, y en concreto con Edublog Enfermería, inicialmente su creación fue rápida, ahora bien el aprovechamiento de la herramienta, aprendizaje en la gestión del mismo, auto-formación a través de tutoriales encontrados en internet, horas de ensayo y error hasta conseguir los resultados deseados,... supuso mayor tiempo de dedicación.

Si los blogs y las redes sociales se pretenden usar con fines académicos, será importante remarcar a los estudiantes las normas éticas, el respeto a la privacidad de los pacientes y seguir las directrices o políticas de medios sociales de la universidad (Skiva, 2011).

Llama la atención los escasos conocimientos sobre el uso de los blogs lo cual indica la necesidad de reforzar la capacitación en esta herramienta. Tras analizarlos, el 48,o \% de los estudiantes tenían conocimientos básicos (entre bajos y muy bajos), sin embargo, después de implementar el Edublog Enfermería para el aprendizaje de la asignatura de $2^{\circ}$ curso, únicamente no lo utilizó el 2,6 \% de los estudiantes. La satisfacción respecto al blog fue muy alta y les resultó de gran utilidad coincidiendo con la investigación de Anderson (2010), quien comprobó que el blog es una herramienta valiosa para el aprendizaje, que permite a los estudiantes una conexión entre la teoría y la práctica profesional y mejora la investigación y colaboración.

Son muchos los autores que enfatizan la necesidad de incorporar al proceso de enseñanza y aprendizaje las herramientas provistas por las tecnologías de la información y comunicación (TIC), entre ellas los blogs (Carley, 2018; Carrillo y Guzmán, 2019; Dapia y Escudero, 2014; Durán, 2011; López, 2009; Williams y Jacobs, 2004). Sin embargo, la mayor parte de las inversiones tecnológicas en materia de educación se destinan a la compra de ordenadores o a la mejora de la calidad de internet. Éstos se utilizan por profesores que, aunque manteniendo enfoques tradicionales de educación, continúan implicándose en proyectos de innovación docente. Las redes sociales, edublogs, webquest, etc., son herramientas de apoyo o un complemento significativo y atractivo pero la calidad educativa no reside en el uso de la tecnología, sino en el proceso de enseñanza y aprendizaje (Levis, 2011; Eger, 2018; Shwu-Fen, Hsiu-Chuan y Ean-Wen, 2017).

Según la Unión Europea, las 21 competencias digitales para los ciudadanos, se organizan en cinco áreas (Vuorikari, Punie, Carretero, y Van den Brande, 2016). Los estudiantes de enfermería de la Universidad de Valladolid poseen un conocimiento 
medio-avanzado en la búsqueda y organización de información a través de la red. Así como en la participación en redes sociales como medio de comunicación con fines lúdicos, siendo necesario reforzar la competencia de creación de contenido digital colaborativo en línea para uso académico de aprendizaje continuo, con el uso de blogs educativos. Además, se debe potenciar sus conocimientos sobre la seguridad de la identidad digital y la resolución de problemas a través de herramientas digitales. Por ello, la creación de un organismo de certificación de la competencia digital para el profesorado universitario (Durán, Gutiérrez, y Prendes, 2016), incentivaría y reconocería los cursos de formación permanente en este ámbito al profesorado, así como la adquisición de dichas competencias, permitiendo ampliar el desarrollo de las competencias digitales de los estudiantes dentro del ámbito universitario renovando así los procesos de enseñanza y aprendizaje.

La Informática educativa, siempre que sea apropiada y crítica, se ha posicionado dentro de las TIC porque favorece el Aprendizaje Colaborativo (Cortés, 2019). Se destacan las ventajas de los blogs y los medios sociales aplicados a la educación en el desarrollo de la creatividad, pues potencian el aprendizaje sin distancias, facilitan la función investigadora, la transferencia de conocimientos y el aprendizaje colaborativo.

Las funcionalidades de interacción en tiempo real y diferido, la colaboración, divulgación de contenidos académicos y científicos dan cuenta de la utilidad y valor de estas herramientas hasta ahora poco utilizadas en la enseñanza universitaria. Se consideran instrumentos para pensar, aprender, conocer y transmitir los conocimientos adquiridos y habría que aprovechar su gran potencial para una educación adaptada a los nuevos estudiantes de enfermería.

Una de las principales limitaciones en este estudio fue el tamaño de la muestra de 225 participantes y su realización en un contexto muy concreto, por ello nuestra aportación sólo permite una aproximación para conocer y describir el perfil sociodemográfico y de conocimiento internauta de los estudiantes y su satisfacción al utilizar un blog, así como el uso de las redes sociales.

Se sugieren futuras investigaciones sobre la utilidad de los blogs y de las redes sociales en la educación en enfermería y en otras disciplinas, contextos o países. Se destaca la necesidad de investigar en esta línea pues el acceso a la información hoy en día es muy fácil no siendo siempre esta fiable. El reto está en que los estudiantes aprendan a clasificar, sintetizar y validar las fuentes informativas con evidencia científica. Además, debe dotarse a los estudiantes de competencias digitales para la comunicación efectiva con un público no especializado que busca en internet gran cantidad de información sobre salud.

El aprendizaje-servicio (ApS) es una manera de aprender presentando un servicio a la comunidad. Por ello, se pueden entender las nuevas tecnologías como uno de los medios de aprendizaje-servicio y que nuestras enfermeras y enfermeros tengan una herramienta más para acercarse y contactar con la población, ejerciendo así su papel como educadores en materia de salud, lo cual es una competencia que 
deben aprender y desarrollar dentro de los planes de estudio del Espacio Europeo de Educación Superior, así como potenciarla durante el desarrollo profesional.

\section{Agradecimientos}

Agradecemos a los estudiantes su participación en este estudio, a los profesionales sanitarios en red por sus valiosas aportaciones y al profesor Dr. Mayo Íscar por su orientación metodológica.

\section{REFERENCIAS}

Adams, S., Brown, M., Dahlstrom, E., Davis, A., DePaul, K., Diaz, V., y Pomerantz, J. (2018). NMC Horizon Report: 2018 Higher Education Edition. Louisville: EDUCAUSE. Recuperado de https:// library.educause.edu/ /media/files/ library/2018/8/2018horizonreport.pdf

Aguaded, J., López, E., y Alonso, L. (2010). Teacher Training and Social Software. Estudios sobre Educación, 18, 97-114. Recuperado de https://dadun.unav.edu/ bitstream/10171/9822/2/ESE 18 5.pdf

Alonso, G. (27 de abril de 2016). Millennials y Generación Z: El gran reto de la educación [Mensaje en un blog]. Recuperado de http://gonzalo-alonso. com/millennials-y-generacion-z-el-granreto-de-la-educacion/

American Library Association. (2013). Digital literacy, libraries, and public policy: Report of the Office for Information Technology Policy's Digital Literacy Task Force. Recuperado de https://www.districtdispatch.org/wpcontent/uploads/2013/01/2012 OITP digilitreport $122 \quad 13 . p d f$

Anderson, S. (2010). Reflective Journaling 2.0: Using Blogs to Enhance Experiential Learning. Journal of Nursing Education, 49(10), 596-596. doi: 10.3928/0148483420100921-02.

Begazo, J. D., y Fernández, W. (2015). Los millennials peruanos: características y proyecciones de vida. Gestión en el Tercer
Milenio, 18(36), 9-15. Recuperado de http://revistasinvestigacion.unmsm.edu. pe/index.php/administrativas/article/ view/11699

Bernal, R. M. (2015). Herramientas telemáticas para la comunicación educativa: Catalogación, análisis y posibilidades de uso de los blogs (Tesis doctoral inédita). Universidad de Murcia, Murcia.

Bloomfield, J. G., Cornish, J. C., Parry, A. M., Pegram, A., y Moore, J. S. (2013). Clinical skills education for graduate-entry nursing students: Enhancing learning using a multimodal approach. Nurse Education Today, 33(3), 247-252. doi: 10.1016/j.nedt.2011.11.009.

Bongarrá, A. C. (2011). Docentes de la "Generación Y". Reflexión Académica en Diseño y Comunicación, 12(15), 169-173. Recuperado de https://fido.palermo.edu/ servicios dyc/publicacionesdc/vista/ detalle publicacion.php?id libro $=269$

Borrás. O. (29 de abril de 2015). Aplicación de las redes sociales en la enseñanza. Web 2.o o web social [Archivo de video]. Recuperado de https://youtu.be/ eTwk7Vl VHU

Carley, S., Beardsell, I., May, N., Crowe, L., Baombe, J., Grayson, A., ... Body, R. (2018). Social-media-enabled learning in emergency medicine: A case study of the growth, engagement and impact of a free open access medical 
education blog. Postgraduate Medical Journal, 94(1108), 92-96. doi: 10.1136/ postgradmedj-2017-135104.

Carrillo, R. L., y Guzmán, A. (2019). Plan estratégico de tecnologías de la información como estrategia universitaria. Avances de investigaciones Grupo Sinergia Digital, 1(1), 61-75. Recuperado de https://uniasturias.edu.co/avancesinvestigaciones-grupo-sinergia-digital/

Carroll, C. L., Bruno, K., y vonTschudi, M. (2016). Social Media and Free Open Acces. Medical Education: The Future of Medical and Nursing Education? American Journal of Critical Care, 25(1), 93-96. doi: 10.4037/ajcc2016622.

Cortés, L. C. (2019). El aprendizaje en línea. Una motivación. Revista iberoamericana de producción académica y gestión educativa, 6(11), 1-8. Recuperado de http://pag.org.mx/index.php/PAG/ article/view/789

Citrome, L. (2015). My two favourite professional social networking sites: LinkedIn and ResearchGate - how they can help you, or hurt you. International Journal of Clinical Practice, 69(6), 623625. doi: 10.1111/ijcp.12681.

Dapia, M., y Escudero, R. (2014). Aprendizaje colaborativo para el uso mediante el edublog en la enseñanza universitaria. Valoración de una experiencia. Enseñanza y Teaching, 32(2), 53-72. doi: 10.14201/ et20143215372.

Dowling, C. (2015). The Connected Generation. Recuperado de https:// www.huffpost.com/entry/the-connectedgeneration b 6117108

Durán, J. (2011). La contribución del edublog como estrategia didáctica. Electronic Journal of Research in Educational of Psychology, 9(23), 331355. Recuperado de http://www.redalyc. org/pdf/2931/293122834016.pdf

Durán, M., Gutiérrez, I., y Prendes, M. P. (2016). Certificación de la competencia TIC del profesorado universitario:
Diseño y validación de un instrumento. Revista mexicana de investigación educativa, 21(69), 527-556. Recuperado de http://www.scielo.org.mx/scielo. php? script $=$ sci arttext $\&$ pid $=$ S1405$\underline{66662016000200527 \& \operatorname{lng}=\mathrm{es} \& t \operatorname{lng}=\mathrm{es}}$

Eger, L., Klement, L., Tomczyk, L., Pisonova, M., y Petrova, G. (2018). Different user groups of university students and their ict competence: evidence from three countries in central Europe. Journal of Baltic Science Education, 17(5), 851-866. Recuperado de http://www. scientiasocialis.lt/ibse/?q=node $/ 708$

Esteve, F., Gisbert, M., y Lázaro, J. (2016). La competencia digital de los futuros docentes: ¿cómo se ven los actuales estudiantes de educación? Perspectiva Educacional, 55(2), 38-54. doi: 10.4151/o7189729-Vol.55-Iss.2-Art.412.

FINE- Europe. (2016). European Federation of Nurse Educators (FINE). Recuperado de http://www.fine-europe.eu/

Frenk, J., Chen, L., Bhutta, Z. A., Cohen, J., Crisp, N., Evans, T.,... Zurayk, H. (2010). Health professionals for a new century: transforming education to strengthen health systems in an interdependent world. The Lancet, 376(9756), 1923-1958. doi: 10.1016/So140- 6736(10)61854-5.

García-Álvarez de Toledo, J., y FernándezSánchez, R. (2011). Difusión y divulgación científica en internet. (G. d. Asturias, Ed.). Asturias: Cienciatec. Recuperado de http://blogs.ujaen.es/cienciabuja/wpcontent/uploads/2013/o6/Difusion-ydivulgacion-cientifica-en-Internet.pdf

Grassley, J. S., y Bartoletti, R. (2009). Wikis and blogs: Tools for online interaction. Nurse Educator, 34(5), 209-213. doi: 10.1097/NNE.obo13e3181b2b59b.

Johnson, L., Adams, S., Cummins, M., Estrada, V., Freeman, A., y Hall, C. (2016). NMC Horizon Report 2016 Higher Education. Austin, Texas: The New Median Consortium. Recuperado de 
http://cdn.nmc.org/media/2016-nmchorizon-report-he-EN.pdf

Kung, Y. (2014). Characteristics of nurses who use social media. Computers, Informatics, Nursing, 32(2), 64-72. doi: 10.1097/CIN.0000000000000033.

Levis, D. (2011). Redes educativas 2.1. Medios sociales, entornos colaborativos y procesos. Revista de Universidad y Sociedad del Conocimiento (RUSC), 8(1), 7-24. doi: 10.7238/rusc.v8i1.965.

López, E. (2009). Innovar con blogs en aulas universitarias. Revista DIM: Didáctica, Innovación y Multimedia, 14, 1-6. Recuperado de http://www. raco.cat/index.php/dim/article/ viewFile/138946/189993

Magg, M. (2005). The potential use of "blogs" in nursing education. Computers, Informatics, Nursing, 23(1), 16-24. doi: 10.1097/00024665-200501000-00005.

Mavrou, K., y Loizou-Raouna, M. (2017). Digital Skills Development and ICT in Inclusive Education: Experiences from Cyprus Schools. Studies in health technology and informatics, 242(1), 828835. doi: 10.3233/978-1-61499-798-6828.

Mayol, J. (2016). La integración del flujo de la tecnología en el flujo del trabajo del profesional sanitario. Recuperado de http://www.comparteinnovacion.philips. es/entornos-saludables/articulos/laintegracion-de-la-tecnologia-en-el-flujode-trabajo-del-profesional-sanitarioparte-1

Molona, P., Valenciano, J., y Valencia-Peris, A. (2015). Los blogs como entornos virtuales de enseñanza y aprendizaje en Educación Superior. Revista Complutense de Educación, 26, 15-31. doi: 10.5209/ rev_RCED.2015.v26. 43791.

Net-Learning (29 de junio de 2016). Webinar Millennials y GenZ: Un desafio para el mundo educativo y laboral [Archivo de vídeo]. Recuperado de https://www. youtube.com/watch?v=HZAob7LxoD4
ONTSI (2016). Los ciudadanos ante la e-sanidad. Ministerio de Industria, Energía y Turismo. Madrid: Ministerio de industria, energía y turismo, Gobierno de España. Recuperado de http://www. ontsi.red.es/ontsi/sites/ontsi/files/los ciudadanos ante la e-sanidad.pdf

Ortega, I., y Soto, I. (2016). Las universidades y la Generación $Z$ [Mensaje en un blog]. Recuperado de https://www. universidadsi.es/las-universidades-lageneracion-z/

Papastavro, E., Hamari, L., Fuster, P., Istomina, N., y Salminen, L. (2016). Using blogs for facilitating and connecting nurse educator candidates. Nurse Education Today, 45(1), 35-41. doi: 10.1016/j. nedt.2016.06.004.

Prensky, M. (2001). Digital Natives, Digital Immigrants. MCB University Press, 9(5), 1-6. doi: 10.1108/10748120110424816.

Prensky, M. (2006). Learning in the Digital Age. Educational Leadership, 63(4), 8-13. Recuperado de http://www.ascd.org/ publications/educational-leadership/ dec05/vol63/numo4/Learning-in-theDigital-Age.aspx

Real Academia Española. (2016). Diccionario de la lengua española (23.a ed.). Recuperado de http://www.rae.es/ rae.html

Redacción Médica. (16 de junio de 2016). De la consulta de enfermería al estrellato en la red. Recuperado de http://www. redaccionmedica.com/la-revista/ reportajes/de-la-consulta-de-enfermeriaal-estrellato-en-la-red-5190

Santillán, A., Cornejo, C., y Ausín, S. (2012). Facebook pages for the broadcasting of media on evidenced-based nursing. Enfermería Global, 11(28), 82-89. Recuperado de http://scielo.isciii.es/pdf/ eg/v11n28/en docencia1.pdf

Schmitt, T., Sims-Giddens, S., y Booth, R. (2012). Social media use in nursing education. The Online Journal of Issues 
in Nurse, 17(3), 2. doi: 10.3912/OJIN. Vol17Noo3Mano2.

Shwu-Fen, C., Hsiu-Chuan, S., y Ean-Wen, H. (2017). The Application of Information and Communication Technology (ICT) in Nursing Education. $\mathrm{Hu}$ li za zhi The journal of nursing, 64(6), 5-11. doi: $10.6224 / J N .000077$.

Skiva, D. (2011). Nursing Education 2.0: The Need for Social Media Policies for Schools of Nursing. Nursing Education Perspectives, 32(2), 126-127. doi: 10.5480/1536-5026-32.2.126.

Verdú, D. (03 de mayo de 2015). La generación $\mathrm{Z}$ cambiará el mundo. El País. Recuperado de http://politica. elpais.com/politica/2015/05/02/ actualidad/1430576024 684493.html

Vuorikari, R., Punie, Y., Carretero, S., y Van den Brande, L. (2016). DigComp 2.o: The Digital Competence Framework for Citizens. Recuperado de: http:// publications.jrc.ec.europa.eu/repository/ bitstream/JRC101254/jrc101254 digcomp\%202.0\%20the\%20digital\%20 competence\%20framework\%20for\%20 citizens.\%20update\%20phase\%201.pdf

Williams, J., y Jacobs, J. (2004). Exploring the use of blogs as learning spaces in the higher education sector. Australasian Journal of Educational Technology, 2O(2), 232-247. doi: 10.14742/ajet.1361.

\section{PERFIL ACADÉMICO Y PROFESIONAL DE LAS AUTORAS}

Carolina González Hernando. Diplomada en Enfermería, Enfermera especialista en Obstetricia y Ginecología; Máster en Investigación Aplicada a la Educación; Máster de Género; Doctora por la UVa (2013). Experiencia profesional: matrona (32 años) y profesora asociada en UVa (10 años). Líneas de investigación: Salud Reproductiva y herramientas Web 2.o en educación para la Salud. IP Proyecto "Blog Mamis y Niños" (premio Buenas Prácticas -Ministerio de Sanidad- (2016)), Premio Sanitaria 2000 (2017) e IP del proyecto Web 2.0 "Edublog Enfermería". Email: carolgh@enf.uva.es

Dirección:

Facultad de Enfermería

Universidad de Valladolid

Avda. Ramón y Cajal, 7, 47005

Valladolid, España

Lorena Valdivieso León. Profesora Ayudante Doctor en el Departamento de Psicología en la Universidad de Valladolid. Doctora Internacional en Psicología (2015) y Master en Investigación Aplicada a la Educación (2011). Líneas de investigación: "Psicología del aprendizaje y de la motivación" y "Estrés académico en estudiantes universitarios". Con numerosas estancias de investigación en universidades de reconocido prestigio internacional, por ejemplo, Universidad de Utrecht. Miembro del Grupo de Investigación de Excelencia (GR179 Castilla y León) en Psicología de la Educación.

Email: lorena.valdivieso@psi.uva.es 
Dirección:

Facultad de Educación, Soria

Universidad de Valladolid

C/Universidad s/n, 42004

Soria, España

Verónica Velasco González. Graduada en Enfermería (2011), Máster en Investigación en Ciencias de la Salud (2011) y en Enfermería Oftalmológica (2016), Doctora con Mención Internacional por la Universidad de Valladolid-UVa- (2016). Profesora Ayudante Doctor en la Facultad de Enfermería de la UVa, Coordinadora del Centro de Lectura del IOBA-UVa y miembro del Centro de Estudios sobre la Seguridad de los Medicamentos-UVa. Líneas de investigación: enfermería, seguridad de los medicamentos, polimorfismos genéticos, retinopatía diabética, telemedicina. Email: veronica.velasco.gonzalez@uva.es

Dirección:

Facultad de Enfermería

Universidad de Valladolid

Avda. Ramón y Cajal, 7, 47005.

Valladolid, España.

Fecha de recepción del artículo: 11/04/2019

Fecha de aceptación del artículo: 01/06/2019

Fecha de aprobación para maquetación: 30/07/2019 